\title{
PREMIUM CALCULATION BY TRANSFORMING THE LAYER PREMIUM DENSITY
}

\author{
By Shaun Wang \\ University of Waterloo, Canada
}

\begin{abstract}
This paper examines a class of premium functionals which are (i) comonotonic additive and (ii) stochastic dominance preservative. The representation for this class is a transformation of the decumulative distribution function. It has close connections with the recent developments in economic decision theory and non-additive measure theory. Among a few elementary members of this class, the proportional hazard transform seems to stand out as being most plausible for actuaries.
\end{abstract}

\section{KEYWORDS}

Premium calculation principle, comonotonicity, stochastic dominance, mean-variance analysis, proportional hazard transform.

\section{INTRODUCTION}

Insurance premium calculation lies at the heart of actuarial science. Traditionally, an insurance risk $X$ is defined as a non-negative loss random variable, and a premium calculation principle refers to a functional $X \mapsto[0, \infty)$. The search for a sound premium calculation principle has been the subject of numerous actuarial papers, and it is still debatable which principle to choose.

In insurance practice, the most widely used method is to base calculation on the first two moments. Since loss distributions are often highly skewed, the first two moments cannot rightly reflect the level of insurance risk. Ramsay (1994) also considered the third moment in his premium calculation formula. Nevertheless, the moment-based method generally violates first order stochastic dominance. The inconsistency of the moment-based methods are discussed by a number of authors (e.g. Venter, 1991; Robbin, 1992).

Besides the moment methods which are commonly used in practice, a few theoretical premium principles are proposed (e.g.Goovaerts et al, 1984). Most of them are rooted in utility theory, for instance, the exponential utility principle (Freifelder, 1979; Gerber, 1979) and the Esscher principle (Bühlmann, 1980). However, Reich (1986) showed that none of these theoretical principles satisfies both the elementary requirements (i) scale-invariance $H(a X)=a H(X)$, and (ii) translation-invariance $H(X+b)=H(X)+b$. 
The Dutch principle (van Heerwaarden and Kaas, 1992) is defined as

$$
H(X)=E[X+\theta \max \{X-\alpha E(X), 0\}], \quad 0 \leq \theta \leq 1 ; \quad \alpha \geq 1 .
$$

It outperforms all previous ones in the sense that it is scale-invariant and translationinvariant. In addition, it preserves second order stochastic dominance. While the risk load component in the Dutch principle has a reinsurance explanation, the relative loading is too restrictive (below 100\%). In practice, the price of a higher layer in casualty insurance may contain a risk load which can be many multiples of the expected loss.

Denneberg (1990) proposed an absolute deviation principle:

$$
H(X)=E(X)+\theta \tau(X), \quad 0 \leq \theta \leq 1,
$$

where $\tau(X)$ is the average absolute deviation from the median. This functional is also scale-invariant and translation-invariant. For an insurance risk with less than $50 \%$ chance of incurring of a claim, this functional coincides with the expected value principle $H(X)=(1+\theta) E(X), \theta \leq 1$, which limits its applicability.

Venter (1991) discussed no-arbitrage implications of insurance pricing. He observed that the only premium principles that preserve layer additivity are those that can be generated from transformed distributions, where the premium for any layer is the expected loss for that layer under the transformed distribution. Based on considerations of layer-additivity, Venter advocated using adjusted distribution principles. Albrecht (1992) showed through an example that not every adjustment to a distribution gives a reasonable premium principle, and he argued that further theoretical development would be needed to arrive at a proper method of adjustment.

Inspired by Venter's insightful observations, Wang (1995) proposed a proportional transform in the hazard rate, or in other words, a power transform in the decumulative distribution function. The proportional hazard transform demonstrates many desirable properties especially in pricing excess-of-loss layers. The present paper was conceived as an attempt to generalize the PH-transform method.

It is the author's intention to take a general axiomatic approach. This paper first settles on the basic requirements for a premium principle, and then searches for premium principles which satisfy these basic requirements. The representation of this class turns out to be a transform of the decumulative distribution function. It includes the PH-transform principle (Wang, 1995) and the absolute deviation principle (Denneberg, 1990) as special cases. This class of premium principles has close connections with the recent developments in (i) economic decision theory under uncertainty and (ii) a mathematical branch concerning non-additive measures.

In section 2 we show that decumulative distribution functions serve a special role as layer net premium densities. In section 3 we examine some desirable properties for a premium functional. In sections 4-6 we discuss a class of premium functionals by transforming the decumulative distribution function. In section 7 we draw connections with non-additive measure theory. In section 8 we discuss the implications in the economics of insurance. 


\section{LAYER NET PREMIUM DENSITY}

An insurance risk $X$ is a non-negative random variable whose distribution is defined by

- the cumulative distribution function $F_{X}(t)=\operatorname{Pr}\{X \leq t\}$, or

- the decumulative distribution function $S_{x}(t)=\operatorname{Pr}\{\mathrm{X}>t\}$.

In general, for a risk $X$, the expected loss can be evaluated directly from its decumulative distribution function:

$$
E(X)=\int_{0}^{\infty} S_{X}(t) d t
$$

Since most insurance contracts contain clauses such as deductible and maximum limit, it is convenient to use a general term of (excess-of-loss) layers.

Definition 1. A layer at $(a, a+h$ ] of a risk $X$ is defined as the loss from an excess-ofloss cover

$$
I_{(a, a+h]}(X)= \begin{cases}0, & 0 \leq X<a \\ (X-a), & a \leq X<a+h \\ h, & a+h \leq X\end{cases}
$$

where $a$ is called the attachment point (retention), and the width $h$ is called the limit.

We simply use notation $I_{(a, a+h)}$, when the risk $X$ is implicitly known.

One can easily verify that the decumulative distribution function for a layer $I_{(a, a+h]}$ is

$$
S_{I_{[a, a+h \mid}}(t)= \begin{cases}S_{X}(a+t), & t<h \\ 0, & t \geq h\end{cases}
$$

The expected loss (or net premium) for a layer at $(a, a+h]$ is

$$
E\left(I_{(a, a+h]}\right)=\int_{a}^{a+h} S_{X}(t) d t
$$

It is noted that $S_{X}(t) d t$ represents the net premium for an infinitesimal layer at $(t, t+$ $d t$. Therefore, the function $S_{X}(t)$ plays an important role as layer net premium density.

The question remains as to how to arrive at risk-adjusted premiums for a risk $X$ or a layer $I_{(a, b]}$. 


\section{DESIRABLE PROPERTIES FOR A PREMIUM FUNCTIONAL}

\subsection{Ordering of risks}

Any premium principle implicitly implies an ordering of preference for all risks. It is a natural requirement for a premium principle to preserve some common ordering of risks. As a simple rule of thumb, higher risks should be associated with higher premiums. In this section, we will introduce some basic concepts of stochastic dominance and apply them to excess-of-loss layers.

\subsubsection{First stochastic dominance}

Definition 2. $X_{1}$ precedes $X_{2}$ under the first stochastic dominance (FSD), if the decumulative distribution function of $X_{1}$ is everywhere lower:

$$
X_{1} \prec_{1 s t} X_{2} \text { if and only if } S_{X_{1}}(t) \leq S_{X_{2}}(t) \text {, for all } t \geq 0 \text {. }
$$

For two layers at $(a, a+h]$ and $(b, b+h]$ of risk $X$, if $a<b$,

$$
S_{X}(a+t) \geq S_{X}(b+t), t \geq 0 \Longrightarrow S_{I_{[a, a+h]}}(t) \geq S_{I_{(b, b+h]}}(t), t \geq 0 .
$$

If a premium principle $H: X \rightarrow[0, \infty)$ preserves FSD, then for $a<b$,

$$
I_{(b, b+h]} \prec{ }_{1 s t} I_{(a, a+h]} \Longrightarrow H\left(I_{(b, b+h]}\right) \leq H\left(I_{(a, a+h]}\right) .
$$

As part of the FSD-preserving requirement, we have:

C1: The absolute premium for layers of a fixed width should decrease at upper layers.

\subsubsection{Second stochastic dominance}

Definition $3 X_{1}$ is less dangerous than $X_{2}\left(X_{1} \prec_{D} X_{2}\right)$ if (i) $E\left(X_{1}\right) \leq E\left(X_{2}\right)$, and (ii) there exists a once-crossing point to such that

$$
\begin{aligned}
& S_{X_{1}}(t) \geq S_{X_{2}}(t) \text { when } t<t_{0}, \\
& S_{X_{1}}(t) \leq S_{X_{2}}(t) \text { when } t \geq t_{0} .
\end{aligned}
$$

The second stochastic dominance (SSD) is a transitive closure of the ordering of dangerousness. It has become a standard definition for a higher risk, partly due to Rothschild and Stiglitz (1970).

Definition $4 X_{I}$ precedes $X_{2}$ under the second stochastic dominance $\left(X_{I} \prec_{2 n d} X_{2}\right)$ if either of the following two equivalent conditions holds (see Kaas et al, 1994):

1. There exists $U_{i}(i=1,2, \ldots, n)$ such that

$$
X_{1} \prec_{D} U_{1}, \quad U_{1} \prec_{D} U_{2}, \ldots, \quad U_{n} \prec_{D} X_{2} .
$$


2.

$$
\int_{x}^{\infty} S_{X_{1}}(t) d t \leq \int_{x}^{\infty} S_{X_{2}}(t) d t, \text { for all } x \geq 0 .
$$

For a risk $X$, consider two layers at $\left(a, a+h_{1}\right]$ and $\left(b, b+h_{2}\right]$ with the same net expected loss, i.e., $E\left(I_{\left(a, a+h_{1}\right]}\right)=E\left(I_{\left(b, b+h_{2}\right]}\right)$. Since the areas underneath the layer net premium density for the two layers are the same, when $a<b$ it is necessary that $h_{1} \leq h_{2}$. In other words, the curve $S_{I_{\left(b, h+h_{2} !\right.}}(t)$ is generally longer and thinner than $S_{I_{\left\{a, a+h_{1}\right]}}(t)$. As in Figure 1, $S_{I_{\left[a, a+h_{1}\right]}}(t)$ and $S_{I_{\left[b, b+h_{2}\right]}}(t)$ cross once at the point $h_{1}$, which implies that the layer at $\left(b, b+h_{2}\right]$ is a higher risk:

$$
I_{\left(a, a+h_{1}\right]} \prec_{D} I_{\left(b, b+h_{2}\right)} .
$$

If a premium principle $H: X \mapsto[0, \infty)$ preserves SSD,

$$
H\left(I_{\left(a, a+h_{1}\right]}\right)<H\left(I_{\left(b, b+h_{2}\right)}\right) \Longrightarrow \frac{H\left(I_{\left(a, a+h_{1}\right]}\right)}{E\left(I_{\left(a, a+h_{1}\right]}\right)}<\frac{H\left(I_{\left(b, b+h_{2}\right]}\right)}{E\left(I_{\left(b, b+h_{2}\right]}\right)} .
$$

As part of the SSD-preserving requirement, we have:

C2: Higher layer should have a higher percentage loading.

\subsection{Comonotonicity}

The concept of comonotonicity was introduced by Yaari (1987) and Schmeidler (1986) and has since been playing a very important role in decision theory under uncertainty.

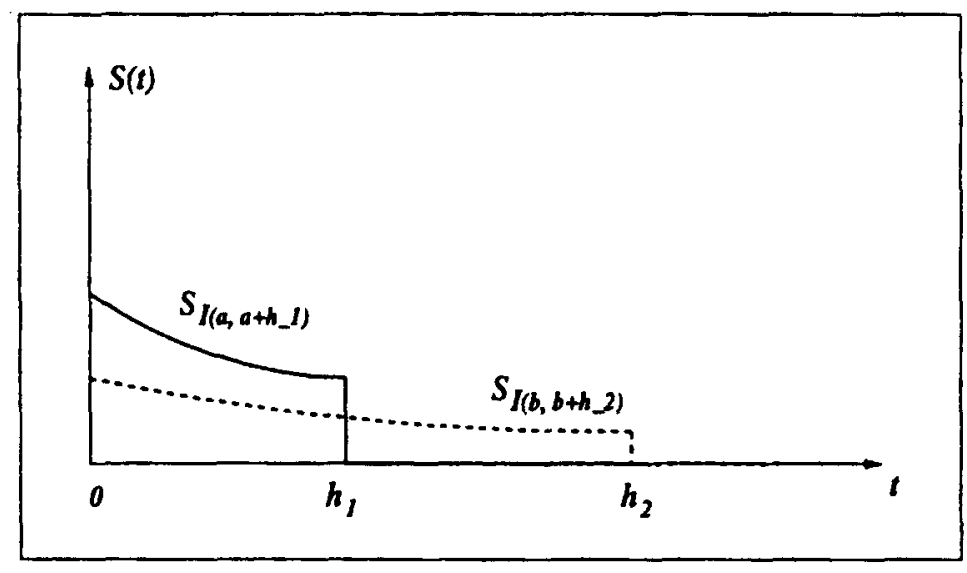

FIGURE 1: Layers with the same expected loss 
Definition $5 X_{1}$ and $X_{2}$ are comonotonic if there exist a risk $Z$ and weakly increasing functions $f, g$ such that $X_{1}=f(Z)$ and $X_{2}=g(Z)$.

Since layers and quota-shares are always increasing functions of the original risk, layers or quota-shares of the same risk are comonotonic. They are bets on the same event and neither of them is hedge against the other.

Now consider a combined policy for two comonotonic risks $X_{1}$ and $X_{2}$. Because of the no-hedge condition, insurers are not willing to give a reduction in the riskload for a combined policy, thus $H\left(X_{1}+X_{2}\right) \geq H\left(X_{1}\right)+H\left(X_{2}\right)$. On the other hand, the maximum premium that insurers can demand for the combined policy is the sum of two individual risk premiums, since otherwise the policy-holder can just buy separate policies, i.e., $H\left(X_{1}+X_{2}\right) \leq H\left(X_{1}\right)+H\left(X_{2}\right)$. To conclude, premiums should be additive for comonotonic risks: $H\left(X_{1}+X_{2}\right)=H\left(X_{1}\right)+H\left(X_{2}\right)$.

As part of the requirement for comonotonic additivity, we have:

C3: Layer premiums should be additive. For any division $0=x_{0}<x_{1}$ $<\ldots<x_{n}<\ldots$,

$$
H(X)=\sum_{i=1}^{\infty} H\left(I_{\left(x_{i-1}, x_{i}\right)}\right)
$$

\section{A CLASS OF PREMIUM PRINCIPLES}

\subsection{Transforming the decumulative distribution function}

Based on the observation that $S_{X}(t)$ is the layer net premium density, a natural idea would be to modify $S_{X}(t)$ to get a 'risk-adjusted' layer premium density $S_{Y}(t)$ :

$$
S_{Y}(t)=g\left[S_{X}(t)\right] .
$$

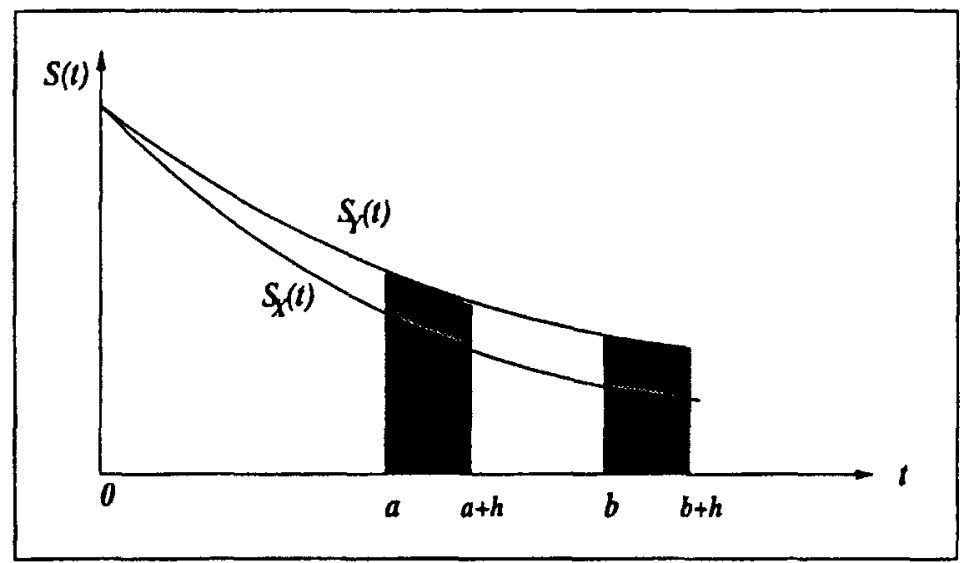

FIGURE 2: An illustration of transforming the layer premium density 
Figure 2 illustrates the basic idea of transforming the decumulative distribution function. The interpretations are as follows:

1. It is necessary to have $H(0)=0$ for a zero risk, which requires $g(0)=0$.

2. The absolute premium for layers of the same width is decreasing if and only if $S_{Y}(t)$ is weakly decreasing. This can be accomplished if, and only if, $g$ is an increasing function.

3. Increased relative loading: When $u=S_{X}(t)$, the relative loading for an infinitesimal layer at $(t, t+d t]$ is

$$
\frac{g\left[S_{X}(t)\right]}{S_{X}(t)}=\frac{g(u)}{u}=\frac{g(u)-0}{u-0},
$$

where the relative loading increases with $t$ if, and only if, $g$ is a concave function of $u$.

4. Layer additivity is automatically satisfied simply because the area underneath the curve $S_{Y}(t)$ is additive.

5. Generally, for a certain loss $\operatorname{Pr}\{X=1\}=1$ we have $H(1)=g(1)$. If we require that $H(1)=1$ then $g(1)=1$, and $S_{Y}(t)=g\left[S_{X}(t)\right]$ defines another decumulative distribution function.

Based on the above observations, we now define a class of premium principles.

Definition 6 For any increasing concave function $g$, with $g(0)=0$ and $g(1)=1$, we define a corresponding premium prmciple:

$$
H(X)=\int_{0}^{\infty} g\left[S_{X}(t)\right] d t
$$

The following properties are straight-forwardly verified:

1. $E(X) \leq H(X) \leq \max (X)$.

2. $H(a X+b)=a H(X)+b, \quad a \geq 0, b \geq 0$.

Since

$$
S_{a X+b}(u)= \begin{cases}1, & 0 \leq u<b \\ S_{X}\left(\frac{u-b}{a}\right), & u \geq b\end{cases}
$$

we have

$$
\begin{aligned}
H(a X+b) & =\int_{0}^{b} 1 d u+\int_{b}^{\infty} g\left[S_{X}\left(\frac{u-b}{a}\right)\right] d u \\
& =b+a \int_{0}^{\infty} g\left[S_{X}(t)\right] d t=a H(X)+b
\end{aligned}
$$

3. Layer additivity: When a risk $X$ is divided into layers at $\left\{\left(x_{i}, x_{i+1}\right], i=0,1\right.$, $\ldots\}$ :

$$
X=I_{\left(0, x_{1}\right)}+I_{\left(x_{1}, x_{2}\right]}+\ldots, \quad 0=x_{0}<x_{1}<x_{2} \ldots
$$


For a layer at $\left(x_{i}, x_{i+1}\right]$, we have

$$
H\left(I_{\left(x_{i}, x_{i+1}\right]}\right)=\int_{x_{1}}^{x_{i+1}} g\left[S_{X}(t)\right] d t
$$

Adding up the layer premiums, we have

$$
\sum_{i=0}^{\infty} H\left(I_{\left(x_{i}, x_{i+1}\right]}\right)=\int_{0}^{\infty} g\left[S_{X}(t)\right] d t=H(X)
$$

4. $H(X)$ preserves the FSD: $X_{1} \prec_{1 s t} X_{2} \Longrightarrow H\left(X_{1}\right) \leq H\left(X_{2}\right)$.

\subsection{Relative loading at higher layers}

Consider the premium principle in (1):

$$
H(X)=\int_{0}^{\infty} g\left[S_{X}(t)\right] d t
$$

where $g$ is an increasing concave function with $g(0)=0$ and $g(1)=1$.

Letting $u=S_{X}(t)$, the relative loading for an infinitesimal small layer at $(t, t+d t]$ is

$$
\phi(t)=\frac{H\left(I_{(t, t+d t]}\right)}{E\left(I_{(t, t+d t]}\right)}=\frac{g\left[S_{X}(t)\right]}{S_{X}(t)}=\frac{g(u)-g(0)}{u-0}
$$

As $t$ increases, $u=S_{X}(t)$ decreases. Since $g(u)$ is concave, $\phi(t)$ is an increasing function of $t$, i.e. increased relative loading at upper layers.

Furthermore, it is easy to see that

$$
\lim _{t \rightarrow \infty} \phi(t)=\lim _{u \rightarrow 0} \frac{g(u)-g(0)}{u-0}=g^{\prime}(0) .
$$

It is noted that the relative loading at upper layers does not exceed $g^{\prime}(0)$.

In property/casualty (re)insurance, the risk load for extremely high layers consists for the most part of the total premium. As observed in Venter (1991), a common phenomenon in reinsurance ratemaking is the minimum rate-on-line, which is the premium divided by the coverage limit. Consider a layer at $(t, t+h]$. Let the width (limit) $h$ be fixed and the attachment point $t$ move to the extreme right tail. Generally the net premium $E\left(I_{(t, t+h]}\right)$ goes to zero as $t$ goes to infinity. If $g^{\prime}(0)$ is finite, the risk-adjusted premium diminishes at the extreme right tail:

$$
H\left(I_{(t, t+h]}\right) \leq g^{\prime}(0) E\left(I_{(t, t+h]}\right) \rightarrow 0, \quad \text { as } t \rightarrow \infty .
$$

Therefore, based on the minimum rate-on-line phenomenon, it may be desirable to have $g^{\prime}(0)=\infty$. 


\subsection{Preserving the SSD}

Theorem 1. The premium principle in (1) preserves the second stochastic dominance:

$$
X_{1} \prec_{2 n d} X_{2} \Longrightarrow H\left(X_{1}\right) \leq H\left(X_{2}\right) .
$$

Proof: We only need to show that $H(X)$ in (1) preserves the ordering of dangerousness: Assume that $E\left(X_{1}\right) \leq E\left(X_{2}\right)$ and there exists $t_{0}$ such that

$$
\begin{aligned}
& S_{X_{1}}(t) \geq S_{X_{2}}(t) \quad \text { when } \quad t<t_{0} \\
& S_{X_{1}}(t) \leq S_{X_{2}}(t) \quad \text { when } \quad t \geq t_{0} .
\end{aligned}
$$

Now we construct a decumulative distribution function:

$$
S_{Z}(t)=\max \left\{S_{X_{1}}(t), S_{X_{2}}(t)\right\}= \begin{cases}S_{X_{1}}(t), & t<t_{0} \\ S_{X_{2}}(t), & t \geq t_{0}\end{cases}
$$

Since the relative loading increases at upper layers, we have

$$
H(Z)-H\left(X_{1}\right) \geq \frac{g\left[S_{X_{1}}\left(t_{0}\right)\right]}{S_{X_{1}}\left(t_{0}\right)} \int_{t_{0}}^{\infty}\left[S_{X_{2}}(t)-S_{X_{1}}(t)\right] d t
$$

and

$$
H(Z)-H\left(X_{2}\right) \leq \frac{g\left[S_{X_{1}}\left(t_{0}\right)\right]}{S_{X_{1}}\left(t_{0}\right)} \int_{0}^{t_{0}}\left[S_{X_{1}}(t)-S_{X_{2}}(t)\right] d t
$$

and the difference (2)-(3) yields

$$
H\left(X_{2}\right)-H\left(X_{1}\right) \geq \frac{g\left[S_{X_{1}}\left(t_{0}\right)\right]}{S_{X_{1}}\left(t_{0}\right)} \int_{0}^{\infty}\left[S_{X_{2}}(t)-S_{X_{1}}(t)\right] d t \geq 0 .
$$

\subsection{Sub-additivity}

In Wang (1995), a proof (due to Ole Hessalager) was given for the sub-additivity of the PH-transform principle. That proof can be generalized to any transform $g$ which is increasing and concave.

Theorem 2. For any two non-negative random variables $U$ and $V$ regardless of dependence, the following inequality holds for the premium principle (1):

$$
H(U+V) \leq H(U)+H(V)
$$

Proof: It is a straightforward generalization of a proof in Wang (1995). 


\subsection{Extra loading for parameter uncertainty}

In practice, actuaries often encounter parameter uncertainty in their modeling. A common method to deal with parameter uncertainty is to use a secondary mixing distribution, that is, to describe the loss distribution as $S_{X}(t \mid \theta)$ where $\theta$ itself has another distribution. It is a desirable property for a premium functional to yield extra risk load for parameter uncertainty. Now we demonstrate that the premium functionals in (1) possess this desirable property.

Applying Jensen's inequality to the concave function $g(z)$, we have

$$
E_{\theta} g\left[S_{X}(t \mid \theta)\right] \leq g\left[E_{\theta} S_{X}(t \mid \theta)\right]=g\left[S_{X}(t)\right] .
$$

Therefore, for the premium principle in (1),

$$
H(X)=\int_{0}^{\infty} g\left[S_{X}(t)\right] d t \geq \int_{0}^{\infty} E_{\theta} g\left[S_{X}(t \mid \theta)\right] d t=E_{\theta} H(X \mid \theta),
$$

with strict inequality unless $\theta$ is degenerative.

As a special case, if $U \neq V$ and $X$ is a mixture:

$$
X= \begin{cases}U, & \text { with probability } a>0 \\ V, & \text { with probability } 1-a>0\end{cases}
$$

then for the premium principle in (1), $H(X)>a H(U)+(1-a) H(V)$.

\subsection{Vertical slicing and comonotonic additivity}

Recall that the net expected loss $E(X)$ is equal to the area below the curve $S_{X}(t)$. While vertical slicing gives $E(X)=\int_{0}^{\infty} S_{X}(t) d t$, horizontal slicing yields that (see Figure 3):

$$
E(X)=\int_{0}^{1} S_{X}^{-1}(q) d q
$$

where the inverse $S_{X}^{-1}(q)$ may not be uniquely defined; however, it does not affect the integration.

One can easily verify that for an increasing function $g$ with $g(0)=0$ and $g(1)=1$,

$$
H(X)=\int_{0}^{\infty} g\left[S_{X}(t)\right] d t=\int_{0}^{1} S_{X}^{-1}(q) d g(q) .
$$

With the aid of horizontal slicing, we can now examine the concept of comonotonicity in terms of layer premium densities.

Lemma 1. If $X$ and $Y$ are comonotonic, then the layer premium densities are additive in the horizontal direction:

$$
S_{X}^{-1}(q)+S_{Y}^{-1}(q)=S_{X+Y}^{-1}(q), \quad 0 \leq q \leq 1
$$




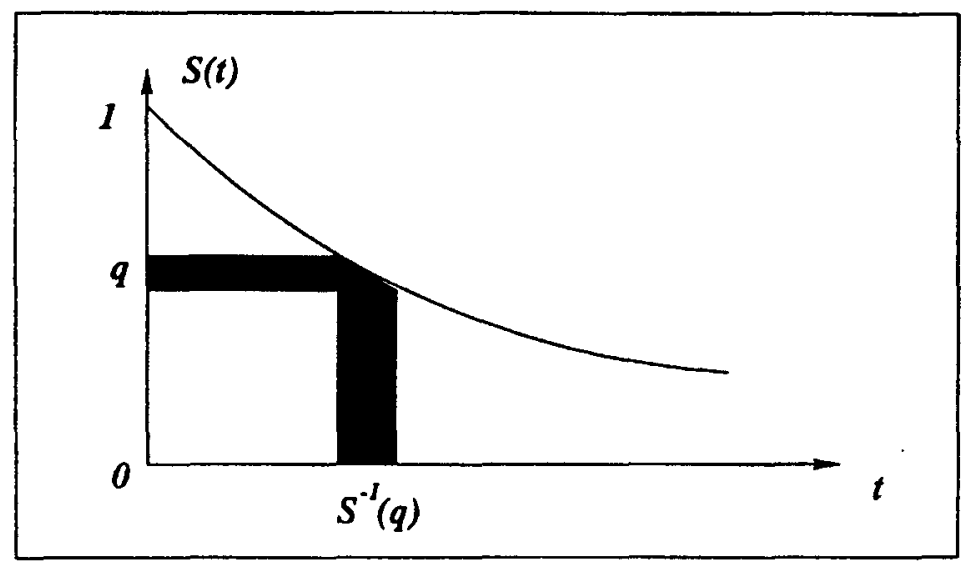

FIGURE 3: Slicing a risk vertically and horizontally

Proof: See Denneberg (1994, p.57; 1990, p.186).

Theorem 3 The premium principle in (1) is additive for comonotonic risks.

Proof: From (4) and Lemma 1 we have

$$
H(X+Y)=\int_{0}^{1} S_{X+Y}^{-1}(q) d g(q)=\int_{0}^{1}\left[S_{X}^{-1}(q)+S_{Y}^{-1}(q) d g(q)=H(X)+H(Y)\right.
$$

\section{SOME ELEMENTARY TRANSFORMS}

In this section we identify some members within the class (1):

$$
H(X)=\int_{0}^{\infty} g\left[S_{X}(t)\right] d t
$$

where $g$ is increasing concave with $g(0)=0$ and $g(1)=1$. We will especially look at some one-parameter families of elementary transforms.

\subsection{Proportional hazard transform}

The PH-transform principle (Wang, 1995) is the simpliest member of the class (1) with

$$
g(x)=\frac{1}{x^{\rho}}, \quad \rho \geq 1 .
$$

It is easy to see that $g^{\prime}(0)=\infty=$ for $\rho>1$. 
In Wang (1995), the following notation is used for the risk-adjusted premium:

$$
\pi_{\rho}(X)=\int_{0}^{\infty}\left[S_{X}(t)\right]^{\frac{1}{\rho}} d t
$$

\subsection{Dual-power functions}

The dual-power transform is a power transform of the cdf:

$$
F_{Y}(t)=\left[F_{X}(t)\right]^{\alpha}, \quad \alpha \geq 1
$$

which implies that

$$
S_{Y}(t)=g\left[S_{X}(t)\right], \quad g(x)=1-(1-x)^{\alpha}, \quad \alpha \geq 1,
$$

where $g$ is increasing concave with $g(0)=0$ and $g(1)=1$.

Since $g^{\prime}(0)=\alpha$, the relative loading at upper layers is bounded by $(\alpha-1)$.

\subsection{Denneberg's absolute deviation principle}

The absolute deviation principle in Denneberg (1990) is equivalent to the following piecewise linear transform $(0 \leq r \leq 1)$ :

$$
g(x)= \begin{cases}(1+r) x, & 0 \leq x<0.5 \\ r+(1-r) x, & 0.5 \leq x \leq 1\end{cases}
$$

When $S_{X}(0)<0.5, H(X)=(1+r) E(X)$, which is the same as the expected value principle.

Since $g^{\prime}(0)=1+r$, the relative loading at upper layers cannot exceed $100 \%$.

\subsection{Quadratic function}

The transform

$$
g(x)=(1+r) x-r x^{2}, \quad 0 \leq r \leq 1
$$

corresponds to the Gini principle which was also discussed in Denneberg (1990).

Since $g^{\prime}(0)=1+r$, the relative loading at upper layers cannot exceed $100 \%$. 


\subsection{Square-root functions}

$$
g(x)= \begin{cases}\frac{\sqrt{1+r x}-1}{\sqrt{1+r}-1}, & r>0 \\ x, & r=0\end{cases}
$$

One can verify that

$$
g^{\prime}(0)=\frac{0.5 r}{\sqrt{1+r}-1}<\infty .
$$

\subsection{Exponential functions}

$$
g(x)= \begin{cases}\frac{1-e^{-\alpha x}}{1-e^{-\alpha}}, & \alpha>0 \\ x, & \alpha=0\end{cases}
$$

One can verify that

$$
g^{\prime}(0)=\frac{\alpha}{1-e^{-\alpha}}<\infty
$$

\subsection{Logarithmic functions}

$$
g(x)= \begin{cases}\frac{\log (1+r x)}{\log (1+r)}, & r>0 \\ x, & r=0\end{cases}
$$

One can verify that

$$
g^{\prime}(0)=\frac{r}{\log (1+r)}<\infty
$$

\subsection{Mixing and composing}

From the elementary transforms one can infinitely construct many other members of the class (1).

Let $g_{i}(i=1,2, \ldots, n)$ be increasing concave functions with $g_{i}(0)=0$ and $g_{i}(1)=1$.

(i) For $p_{i} \geq 0$ with $\sum_{i=1}^{n} p_{i}=1$, the function $g=\sum_{i=1}^{n} p_{i} g_{i}$ is also an increasing concave function with $p(0)=0$ and $g(1)=1$.

(ii) The function $g(u)=g_{2}\left[g_{1}(u)\right]$ is also an increasing concave function with $g(0)=0$ and $g(1)=1$. 
By mixing and composing, one can get some two-parameter families of premium principles. For instance, $H(X)=\theta E(X)+(1-\theta) \pi_{\rho}(X),(0 \leq \theta \leq 1)$.

\subsection{Remarks}

(i) Among all the above elementary transforms, only the PH-transform has $g^{\prime}(0)=\infty$. This special feature will become important in the performance test in the next section.

(ii) In the definition 6 , one can allow $g(1) \neq 1$, except for the property $E(X) \leq H(X) \leq \max (X)$, all other properties still hold. A simple example is $H(X)=c \pi_{\rho}(X),(c>0)$.

\section{A PERFORMANCE TEST}

\subsection{Mean-variance analysis}

The main-stream definition for a higher risk is based on FSD or SSD. Since all the premium principles defined in (1) preserve FSD and SSD, we can now use some other criteria, namely the mean-variance (or higher moments) analysis, which has been widely used in finance and insurance risk theory.

Example 1: Consider the following two risks:

$$
\begin{aligned}
& S_{U}(t)= \begin{cases}\frac{1}{4}, & 0 \leq t<4, \\
0, & 4<t .\end{cases} \\
& S_{W}(t)=\left(\frac{1}{1+t}\right)^{2} \quad \text { (two - point) }
\end{aligned}
$$

where both have the same mean $(=1)$. While the two-point risk $U$ has a finite variance (=3), the variance for the Pareto risk $W$ is infinite.

By calculating the layer net premiums $E\left(I_{(x, \infty)}\right)$ one can see that there is no SSD ordering between the the two-point risk $U$ and the Pareto risk $W$.

However, according to the popular mean-variance analysis, the Pareto risk is a higher risk since it has an infinite variance. Most insurers would share the same view that the Pareto risk $W$ is more risky than the two-point risk $U$.

In fact, a deeper theoretical analysis (Kaas et al, 1994, p. 50-53) shows that every regular decision-maker who has a smooth (to be precise, twice differentiable) increasing concave utility function perceives that the Pareto risk is a higher risk than the two-point risk. 


\subsection{Comparison of performance}

To compare the performance of various elementary transforms given in the preceding section, we apply the premium principles to the two-point risk $U$ and the Pareto risk $W$ in Example 1. We choose the parameter values such that $H(U)=1.3$ for all the premium principles.

From Table 1, one can see that only the PH-transform gives the Pareto risk $W$ a premium higher than 1.3. (Recall that only the PH-transform has $g^{\prime}(0)=\infty$ )

For the PH-transform principle, we have

$$
\pi_{\rho}(U)=4^{1-\frac{1}{\rho}}, \quad \pi_{\rho}(W)= \begin{cases}\frac{\rho}{2-\rho}, & \rho<2 \\ \infty, & \rho \geq 2\end{cases}
$$

For any $\rho>1$, the risk-adjusted premium always assigns a higher premium to the Pareto risk (see Figure 4).

Table 1. Premiums for the two-point risk $U$ and the Pareto risk $W$

\begin{tabular}{|l|l|l|l|}
\hline PRINCIPLE & PARAMETER & $\mathrm{H}(\mathrm{U})$ & $\mathrm{H}(\mathrm{W})$ \\
\hline PH-transform & $\rho=1.233$ & 1.3 & 1.608 \\
\hline Square-root & $r=3.157$ & 1.3 & 1.2903 \\
\hline Logarithmic & $r=1.055$ & 1.3 & 1.2782 \\
\hline Exponential & $\alpha=.7594$ & 1.3 & 1.2708 \\
\hline Quadratic & $r=.4$ & 1.3 & 1.2667 \\
\hline Dual-power & $\mathrm{r}=1.366$ & 1.3 & 1.2662 \\
\hline Denneberg & $r=.3$ & 1.3 & 1.2485 \\
\hline
\end{tabular}

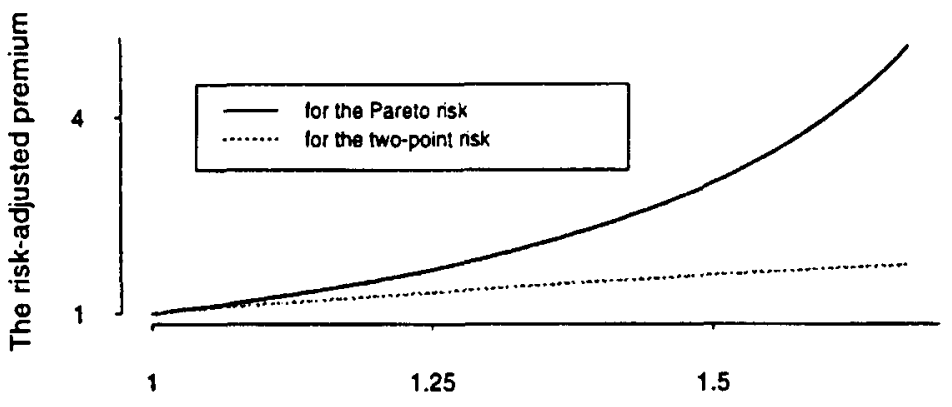

The PH-transform parameter ... tho

FIGURE 4: PH-transform always gives a higher premium to the Pareto risk 


\subsection{A remark}

We have seen that all the elementary transforms with $g^{\prime}(0)<\infty$ yield a lower premium to the Pareto risk, which confirms an earlier observation that, when $g^{\prime}(0)$ is finite, the relative loading does not increase fast enough at upper layers.

A necessary condition for $H(U)<H(W)$ seems to be that $g^{\prime}(0)=\infty$. However, $g^{\prime}(0)=\infty$ is not a sufficient condition for $H(U)<H(W)$. For exemple, consider the transform

$$
g(x)=\left[\sin \left(\frac{\pi}{2} x\right)\right]^{0.95},
$$

where $g$ is in family (1) with $g^{\prime}(0)=\infty$. However, $H(U)=1.606>H(W)=1.588$.

\section{A NON-ADDITIVE MEASURE REPRESENTATION}

The class of premium principle (1) has close connections with the recently developed non-additive measure theory. Denneberg's (1994) monograph serves as an execellent reference on this subject.

Let $P$ be a probability measure on a $\sigma$-algebra $2^{\Omega}$ and $g:[0,1] \rightarrow[0,1]$ be an increasing concave function with $g(0)=0$ and $g(1)=1$. The set function $\mu=g \circ P$ defines a distortion of the underlying probability measure $P$ and has been discussed in a number of contexts (e.g., Denneberg, 1994, p.17). In fact, $\mu$ is a sub-additive measure and the premium functional

$$
H(X)=\int X d \mu=\int_{0}^{\infty}(g \circ P)\{X>t\} d t=\int_{0}^{\infty} g\left[S_{X}(t)\right] d t
$$

is a Choquet intergral of $X \geq 0$.

\subsection{A representation theorem}

In the non-additive measure theory the following representation theorem holds (Denneberg, 1994, p.144):

Theorem 4 Let $\boldsymbol{B V}$ be an appropriate set of bounded random variables. If functional $\mathrm{H}: \boldsymbol{B} \boldsymbol{V} \rightarrow[0, \infty)$ is (i) comonotonic additive, (ii) preserving $F S D$, and (iii) $H(1)=1$, then there exists a distortion function $g$ such that

$$
H(X)=\int_{0}^{\infty}(g \circ P)\{X>t\} d t=\int_{0}^{\infty} g\left[S_{X}(t)\right] d t
$$


Furthenmore, $H(X+Y) \leq H(X)+H(Y)$ if and only if $g$ concave.

As a pure mathematical result (in different forms), Theorem 4 was first proved by Schmeidler (1986). It is noted that for bounded variables, the sub-additivity Theorem 2 becomes a corollary of Theorem 4 ; however, our Theorem 2 also holds for unbounded variables.

When translated back to our language of premium principles, Theorem 4 says that transforming the decumulative distribution function is the only way to get comonotonic-additive and SSD-preserving premium principles (as we have seen in the illustration in section 4.1). The class (1) gives a definition and also a representation for all comonotonic-additive and SSD-preserving premium functionals.

\subsection{Another look at actuarial literature}

This representation theorem rules out most traditional premium principles. Among a dozen premium principles discussed in Goovaerts et al (1984), none (except the net premium) has representation (1).

The Dutch principle does not have representation (1), since layer-additivity is violated. For an example, let

$$
\operatorname{Pr}\{Y=0\}=\operatorname{Pr}\{Y=1\}=\operatorname{Pr}\{Y=2\}=\frac{1}{3} .
$$

Consider a special case of the Dutch principle with $\alpha=1$ and $\theta=1$ :

$$
H(X)=E(X)+E\left(I_{(E(X), \infty]}\right) .
$$

We have $H(X)=1 \frac{1}{3}$ but on the other hand

$$
H\left(I_{(0,1]}\right)+H\left(I_{(1,2]}\right)=\left(\frac{2}{3}+\frac{2}{3} \times \frac{1}{3}\right)+\left(\frac{1}{3}+\frac{1}{3} \times \frac{2}{3}\right)=1 \frac{4}{9}>H(X) .
$$

Venter (1991) has shown that every premium principle which satisfies layeradditivity comes from an adjusted distribution principle. He also gave some examples: (i) transforming the probability density function $f(z)$ to $a f(a x)$ (which is a scale transform); and (ii) multiplying the density $f(x)$ by a non-negative function $h(x)$ (a special case of which is the covariance principle). However, not every layeradditive principle preserves the FSD. Albrecht (1992) gave an example to show that a scale transform can lead to a price below the expected cost for the retained portion below a franchise deductible.

From Theorem 4 it becomes evident that in order to have both layer-additivity and the preserving of FSD, one has to transform directly the decumulative distribution function. This would rule out the scale-transform and the covariance principle. It is noted that any transform $Y=g(X)$ would lead to $S_{Y}(t)=S_{X}\left[g^{-1}(t)\right]$, which modifies the function $S_{X}(\cdot)$ from inside, thus not a direct transform of the decumulative distribution function. 


\section{ECONOMICS OF INSURANCE}

\subsection{Expected utility theory: an old time religion}

Utility theory was introduced to insurance economics by Borch (1961) and since then it has been playing a dominant role. Given initial wealth $\omega_{0}$ and an agent's utility function $u$, the premium that the agent is willing to pay (or accept) for insuring risk $X$ can be derived from the expected utility equation:

$$
u\left(\omega_{0}-P\right)=E\left[u\left(\omega_{0}-X\right)\right] .
$$

Within this conceptual framework, a number of premium principles have been developed by European actuaries (see Goovaerts et al, 1984); for instance, the exponential utility principle. The widely-used variance principle $P(X)+\alpha \operatorname{Var}(X)$ can be viewed as an approximation from the expected utility equation by using the first two terms of Taylor expansion.

Embedded in the expected utility theory are some precise orderings of preference. The first stochastic dominance is the common ordering shared by all decisionmakers with increasing utility functions. The second stochastic dominance is the common ordering shared by all decision-makers with increasing concave utility functions. It is this precise ordering of preference that makes expected utility theory a very popular conceptual framework.

Let the symbol $\succ$ and $\sim$ represent strict preference and indifference, respectively. As for Euclidean geometry, the utility theory is based on five axioms (e.g. Fishburn, 1982):

EU.1 If prospects $V_{1}$ and $V_{2}$ have the same cumulative distribution function, then $V_{1}$ $\sim V_{2}$.

EU.2 Weak order: $\succeq$ is reflective, transitive and connected.

EU. $3 \succeq$ is continuous in the topology of weak convergence.

EU.4 preserves the FSD.

EU.5 If $S_{V_{1}} \succeq S_{V_{2}}$, for and any $p \in[0,1]$, the probabilistic mixture satisfies:

$$
\left[(1-p) S_{V_{1}}+p S_{V_{2}}\right] \succeq\left[(1-p) S_{V_{2}}+p S_{V_{3}}\right]
$$

From the above five axioms of preference, one can show the existence of a utility function $u$ such that ' $V_{1} \succ V_{2}$ if and only if $E\left[u\left(V_{1}\right)\right] \succeq E\left[u\left(V_{2}\right)\right]$.'

The axiomatic foundation makes many people believe that utility theory is the only legitimate theoretical tool for analyzing decision under uncertainty, which itself explains its dominant role in the economics of insurance.

While economists are mainly concerned with the ordering of preference, actuaries are interested in concrete numbers in real terms (dollars, pounds, etc). Unfortunately, the expected utility paradigm does not promise a consistent premium principle (e.g. Reich, 1986). In fact, the layer-additivity only holds for linear utility functions. 


\subsection{Yaari's dual theory of choice under risk}

As with the Euclidean axiom system, a number of people challenged the fifth axiom in the expected utility theory (e.g. Allais, 1953; Machina, 1982). Quiggin (1982) proposed an anticipated utility theory which does not follow the expected utility axioms.

In an independent approach, Yaari (1987) proposed an alternative to the fifth EU axiom:

EU.5* If $V_{1}, V_{2}$ and $V_{3}$ are comonotonic and $V_{1} \succeq V_{2}$, for any $p \in[0,1]$, the outcome mixture satisfies: $(1-p) V_{1}+p V_{3} \succeq(1-p) V_{2}+p V_{3}$.

By replacing the fifth axiom EU.5 by EU.5*, Yaari (1987) developed a dual theory of choice under risk, which turns out to be a special case of Quiggin (1982). Yaari's axiomatic approach breaks the old belief that the expected utility paradigm is the only legitimate theoretical tool for analyzing decision under uncertainty.

Under axiom EU.1-4 \& EU.5*, Yaari (1987) showed that there exists a dual utility function $h:[0,1] \rightarrow[0,1]$ such that a certain equivalent to a random economic prospect $V$ on interval $[0,1]$ is

$$
\int_{0}^{1} h\left[S_{V}(t)\right] d t
$$

where the function $h$ is convex when an agent is risk-averse.

Now assume that an agent has initial wealth 1 and is facing a random loss $X$ on the interval $[0,1]$. By facing risk $X$, the agent's economic prospect is $V=1-X$. Let $P$ be the insurance premium for risk $X$. Analogous to the expected utility equation, we have a certain-equivalent relation:

$$
1-P=\int_{0}^{1} h\left[S_{1-X}(t)\right] d t .
$$

Since $S_{1-X}(t)=\operatorname{Pr}\{X<1-t\}$, we have

$$
\begin{aligned}
1-P & =\int_{0}^{1} h\left[1-S_{X}(1-t)\right] d t=\int_{0}^{1} h\left[1-S_{X}(z)\right] d z ; \\
P & =\int_{0}^{1} g\left[S_{X}(z)\right] d z, \quad \text { where } g(x)=1-h(1-x) .
\end{aligned}
$$

One can verify that $g$ is concave if and only if $h$ is convex.

As a special case of Yaari's equation $(5)$, if $h(x)=1-(1-x)^{\frac{1}{\rho}}$, we get $g(x)=x^{\frac{1}{\rho}}$ which corresponds to the PH-transform in Wang (1995). It is noted that Yaari's axiomatization is valid for uniformly bounded random variables, whereas the PH-transform premium functional is defined also for unbounded random variables. (A referee pointed out that the continuity axiom may have to be weakened or modified in order to include unbounded random variables). 


\subsection{Economic interpretations}

Firstly we need to distinguish between two types of risk-aversion:

- Risk-aversion-l: the traditional utility theory expresses as an attitude towards wealth, in the mean while it is linear with respect to probability.

- Risk-aversion-2: The proposed premium principle (1) describes as an attitude towards probabilities (uncertainty), in the mean while it is linear with respect to wealth.

When interpreting the premium principle (1), we wish to distinguish individual insurers and the whole insurance industry.

- An insurer by itself can only take on limited liability. If the loss is small scale relative to its financial capacity, the insurer exhibits local risk-aversion-2. However, at relatively large loss scales, an insurer may start to exhibit riskaversion-1. Even though the threshold for this transition may not be clear-cut, it may be modeled by a utility function or by using a maximum variance constraint.

- The whole insurance industry, as a collective of individual companies competing in the insurance market, cannot feel 'pain' as a result of any single loss, so it cannot be risk-averse-1. On the other hand, the insurance industry is in a business to make a profit. We can say that 'profit-seeking' is a hidden name for being 'risk-averse-2'.

- Individual insurers are price-takers, not price-makers (Meyers, 1991). The premium principle (1) may provide a model for market premiums.

Regarding additivity, we have the following comments.

- Traditional variance principle and exponential utility principle are additive for independent risks but super-additive for comonotonic risks.

- By contrast, the premium principle (1) is additive for comonotonic risks but strictly sub-additive for independent risks.

- The strict sub-additivity for independent (or less correlated) risks explains the mechanism for risk exchange. However, one has to admit that the insurance market is not as efficient as the financial market. Significant transaction costs may discourage many theoretically justifiable risk-exchanges.

- As an operational rule, insurers may apply the premium principle (1) to individual risks rather than to the collective claim distribution. In which case, additivity holds for individual risks.

- Theoretically, suppose that transaction costs are negligible, the optimal strategy is to form cross-hedging for all diversifiable risks (e.g. form a loss index of all companies combined). The remaining risks (and their layers or quota-shares) are all comonotonic. The premium principle (1) is additive for comonotonic risks. 


\subsection{Conclusion}

In this paper we have discussed a class of premium principles defined by

$$
H(X)=\int_{0}^{\infty} g\left[S_{X}(t)\right] d t
$$

where $g$ is increasing concave with $g(0)=0$ and $g(1)=1$. The premium principle $H(X)$ is comonotonic-additive and preserves the second stochastic dominance. Its close connections with the recent developments in economic decision theory may make it more justifiable. In order to get unbounded relative loading at upper layers, as supported by the minimum rate-on-line phenomenon in reinsurance ratemaking, an additional requirement is that $g^{\prime}(0)=\infty$. After investigating a number of elementary transforms, the PH-transform seems to stand out as the most plausible method for actuaries.

\section{ACKNOWLEDGMENTS}

Part of this paper was presented at the Casualty Actuaries Reinsurance Seminar in New York, June 26-27, 1995. The completion of this work was supported by an AERF/CKER joint grant. The author thanks Harry Panjer, Gary Venter and an anonymous referee for comments and suggestions.

\section{REFERENCES}

Albrecht, P. (1992). Premium calculation without arbitrage? A note on a contribution by G. Venter. ASTIN Bulletin, 22, 247-254.

AllaIS, M. (1953). Le comportement de l'homme rationel devant le risque: critique des axioms et postulates de l'ecole Americaine. Econometrica 21(4), 503-46.

BorCH, K.H. (1961). The utility concept applied to the theory of insurance. ASTIN Bulletin, 1, 170-191.

BuhlmanN, H. (1980) An economic premium principle. ASTIN Bulletin, 11, 52-60.

Denneberg, D. (1990). Premium calculation: why standard deviation should be replaced by absolute deviation. ASTIN Bulletin, 20: 181-190.

DenNeberg, D. (1994). Non-Addititue Measure And Integral. Kluwer Academic Publishers, Boston.

Fishburn, P. (1982). The Foundations of Expected Utility. Reidel, Dordrecht, Holland.

FreifeldER, L.R. (1979). Exponential utility theory ratemaking: an alternative ratemaking approach. Journal of Risk and lnsurance, 46: 515-530.

Gerber, H.U. (1979). An Introduction to Mathematical Risk Theory. Huebner Foundation Monograph, Wharton School, University of Pennsylvania.

Goovaerts, M.J., DE Vylder, F. AND HaEzendonck, J. (1984) Insurance Premiums: Theory and Applications. North-Holland, Amsterdam.

Kaas, R., Van Heerwaarden, A.E., And Goovaerts, M.J. (1994) Ordering of Actuarial Risks. Ceuterick, Leuven, Belgium.

Machina, M. (1982) 'Expected Utility' analysis without the independence axiom. Econometrica, 50(2), 217-323.

Meyers, G.G. (1991). The competitive market equilibrium risk load formula for increased limits ratemaking. Proceedings of the Casualty Actuarial Society. Vol. LXXVIII, 163-200.

QUIGGIN, J. (1982). A theory of anticipated utility. Journal of Economic Behavior and Organization, 3(4), 323-43. 
RAMSAY, C.M. (1994) Loading gross premiums for risk without using utility theory, with Discussions. Transactions of the Society of Actuaries. Vol. XLV, p. 305-349.

ReICH, A. (1986) Properties of premium calculation principles. Insurance: Mathematics and Economics, 5. 97-101.

RoBBIN, (1992). Discussion of Meyers' paper - The competitive market equilibrium risk load formula for increased limits ratemaking. Proceedings of the Casualty Actuarial Society. Vol. LXXIX, 367-384.

Rotschield, M. AND STIGLITZ, J.E. (1970) Increasing risk I: a definition. Journal fo Economic Theory 2 , 225-243.

SCHMeIder, D. (1986). Integral representation without additivity. Proceedings of the American Mathematical Society, 97: 225-261.

VAN HeERWAarden, A.E. AND KaAs, R. (1992) The Dutch premium principle. Insurance: Mathematics and Economics, 11, 129-133.

VENTER, G.G. (1991) Premium calculation inmplications of reinsurance without arbitrage. ASTIN Bulletin, 21, 223-230.

WANG, S. (1995) Insurance Pricing And Increased Limits Ratemaking By Proportional Hazards Transforms. Insurance: Mathematics and Economics, 17, 43-54.

YAARI, M.E. (1987). The dual theory of choice under risk. Econometrica, 55: 95-115.

ADDRESS OF THE AUTHOR:

Dept. of Statistics and Actuarial Science

University of Waterloo

Waterloo, ON N2L $3 G 1$ Canada

E-mail: sswang@math.uwaterloo.ca 